DOI: 10.20472/IAC.2017.032.008

\author{
KUO-HSUAN CHIN \\ Department of Economics, Feng Chia University, Taiwan
}

XUE LI

Department of Economics, Institute of Chinese Financial Studies, Southwestern University of Finance and

Economics, China

\title{
BAYESIAN FORECAST COMBINATION IN VAR-DSGE MODELS
}

\begin{abstract}
:
We evaluate the performance of the individual and combination forecasts in the estimated Bayesian VARs with economic and non-economic information. Specifically, we conduct an out-of-sample forecasting experiment in the model with statistical and/or DSGE priors over the time period before and after the financial crisis. In the most of cases, we obtain the unbiased forecasts of the interest rate but the biased forecasts of output growth and inflation rates under the unbiasedness test. In particular, we find the estimation of Bayesian VARs with economic information about the financial friction is helpful to improve the forecasting performance of the interest rate, evaluated in terms of the modified DM test, point and density forecasts. Moreover, the combination forecasts of the interest rate generated from the model with both statistical and DSGE priors are unbiased, and they also perform better than the combination or the individual forecasts generated with only statistical priors at statistically significant level of $5 \%$. The selection of the weighting-scheme in forecast combination, adopting equal weights for the simple average or the log predictive likelihoods in Bayesian model averaging, is irrelevant to the conclusion made above.
\end{abstract}

\section{Keywords:}

Bayesian Model Averaging, DSGE-VAR, Financial Friction, Forecast Combination.

JEL Classification: E37, E44, E47 